\title{
Thyrotoxic Periodic Paralysis in a Nigerian Male
}

\author{
A Vogazianou*, ${ }^{*}$, T O’Shea§, P Hyatt ${ }^{\S}$, C Nethaji ${ }^{\S}$, A Garg $\$$
}

*Department of Endocrinology \& Diabetes Mellitus, University College London Hospital

${ }^{\S}$ Department of Endocrinology and Diabetes Mellitus, North Middlesex University Hospital

\section{North Middlesex University Hospital W/HS \\ NHS Trust}

\section{INTRODUCTION}

Periodic paralysis is a rare condition, characterised by muscle weakness in the presence of triggers including cold, heat, high carbohydrate meals or physical activity. It is classically described in the presence of profound hypokalaemia; when this occurs in conjunction with thyrotoxicosis it is termed thyrotoxic periodic paralysis.

\section{CASE HISTORY}

A 30 year old Nigerian male presented to the Emergency Department with a three hour history of inability to move. He reported similar episodes over the preceding two years, for which he had not sought medical attention. He had associated severe muscle cramps in his upper and lower limbs.

On examination he was noted to have profound proximal myopathy. He had a moderate goitre which was smooth to palpation. A loud bruit was audible over the thyroid. There was no retrosternal extension, palpable nodules or neck lymphadenopathy. He had a sinus tachycardia and was markedly tremulous. He reported no family history of thyroid disease.

\section{INVESTIGATIONS AND MANAGEMENT}

Biochemical Investigations

-Serum Potassium (K) 2.0mmol/L (3.5-5.5)

$\cdot \mathrm{TSH}<0.01 \mathrm{mU} / \mathrm{L}(0.35-5.5)$

-fT4 72.7pmol/L (10.0-22.7)

-Creatinine Kinase 2047U/L (<170)

-TSH-Receptor Antibodies 28.17U/L (0-0.4)
He was treated with intravenous potassium replacement in the Emergency Department, with rapid resolution of his paralysis and myopathy. He was commenced on propranolol and carbimazole for treatment of Grave's Disease. On discharge his potassium was $5 \mathrm{mmol} / \mathrm{L}$ without additional supplementation. Since discharge he has remained euthyroid, and has had no further episodes of paralysis. His creatinine kinase has normalised.

\section{DISCUSSION}

Thyrotoxic periodic paralysis (TPP) is a rare complication of hyperthyroidism, which has been reported, predominantly, in Asian patients ${ }^{1}$. Whilst it has also been reported in approximately $2 \%$ of Chinese and Japanese patients with hyperthyroidism, it appears to only occur sporadically in other populations. It occurs more frequently in males (with approximately an 11:1 male:female predominance $^{2}$. It is most common in 20-40 year olds and presents as recurrent episodes of myopathy which is more marked proximally. It can be life threatening: due to ventricular arrhythmias or respiratory muscle compromise ${ }^{3}$ (which, however, are rare).

It has been associated with all forms of hyperthyroidism; its occurrence is not isolated to autoimmune thyroid disease ${ }^{4}$. It is characterised by recurrent episodes of paralysis in the presence of both hypokalaemia and thyrotoxicosis. The thyrotoxicosis may be mild or subclinical. Hypokalaemia occurs due to intracellular shift of potassium rather than true potassium depletion. It is likely due to increased sodium-potassium adenosine triphosphate pump activity (Na-K ATPase), which may be upregulated due to the direct effects of thyroid hormone or indirectly due to sympathetic overactivity ${ }^{5}$. Patients with TPP also appear to have an exaggerated insulin response to glucose/carbohydrate ingestion. Carbohydrate heavy meals have been noted as a trigger for episodes of paralysis, and it has been suggested that hyperinsulinaemia may play a role in Na-K ATPase activation ${ }^{6}$.

TPP has been increasingly reported in non-Asian patients in recent years, but is very rarely found in black African patients. It is important that it be considered in all patients presenting with episodes of hypokalaemia and paralysis/myopathy regardless of ethnicity. Treatment of thyrotoxicosis is paramount and is associated with complete resolution of episodes of paralysis.

\section{REFERENCES}

Kung, Clinical review: Thyrotoxic periodic paralysis: a diagnostic challenge, J Clin Endocrinol Metab. 2006 Jul;91(7):2490-5. Epub 2006 Apr 11.

Ober KP 1992 Thyrotoxic periodic paralysis in the United States. Report of 7 cases and review of the literature. Medicine 71:109-120

Liu YC, Tsai WS, Chau T, Lin SH 2004 Acute hypercapnic respiratory failure due to thyrotoxic periodic paralysis. Am J Med Sci 327:264-267

Tinker TD, Vannatta JB 1987 Thyrotoxic hypokalemic periodic paralysis: report of four cases and review of the literature. J Okla State Med Assoc 80:76-83

Chan A, Shinde R, Chow CC, Cockram CS, Swaminathan R 1991 In vivo and in vitro sodium pump activity in subjects with thyrotoxic periodic paralysis. Br Med J 303:1096-1099

Lee KO, Taylor EA, Oh VMS, Cheah JS, Aw SE 1991 Hyperinsulinaemia in thyrotoxic hypokalaemic periodic paralysis. Lancet 337:1063-1064 